\title{
Rational Approximations for the Fresnel Integrals
}

\author{
By Mark A. Heald
}

\begin{abstract}
A class of simple rational polynomial approximations for the Fresnel integrals is given with maximum errors from $1.7 \times 10^{-3}$ down to $4 \times 10^{-8}$. The domain $[0, \infty]$ is not subdivided. The format is particularly convenient for programmable hand calculators and microcomputer subroutines.
\end{abstract}

The best-known algorithms for computer evaluation of the Fresnel integrals,

$$
C(x)+i S(x)=\int_{0}^{x} \exp \left(i \frac{1}{2} \pi t^{2}\right) d t
$$

are those of Hastings [1], [4] and of Boersma [2]. The former is of very limited precision. The latter is relatively cumbersome and is subject to nonobvious underflow (subtraction) errors, as is direct evaluation of the standard Maclaurin and asymptotic series. An extensive set of rational polynomial approximations has been given by Cody [3]. These are efficient for very high-precision work, but tedious for intermediate levels of precision because the domain is subdivided into five intervals.

Our approach follows Hastings but recasts the formulas into the polar form:

$$
\begin{aligned}
& C(x)=\frac{1}{2}-R_{l m}(x) \sin \left[\frac{1}{2} \pi\left(A_{j k}(x)-x^{2}\right)\right], \\
& S(x)=\frac{1}{2}+R_{l m}(x) \cos \left[\frac{1}{2} \pi\left(A_{j k}(x)-x^{2}\right)\right],
\end{aligned}
$$

where the $R_{/ m}$ and $A_{j k}$ functions are rational approximations of the form

$$
\begin{aligned}
R_{l m} & =\sum_{i=0}^{l} c_{i} x^{i} / \sum_{i=0}^{m} d_{i} x^{i}, \\
A_{j k} & =\sum_{i=0}^{j} a_{i} x^{i} / \sum_{i=0}^{k} b_{i} x^{i} .
\end{aligned}
$$

The domain of $x$ is positive real numbers from zero to infinity.

We consider error functions of the form

$$
\begin{gathered}
\delta R=R_{l m}-R_{0}, \\
\delta A=\frac{1}{2} \pi R_{0}\left(A_{j k}-A_{0}\right),
\end{gathered}
$$

where $R_{0}$ and $A_{0}$ are exact values. These functions represent orthogonal errors in the plane of the Cornu spiral, with the coefficient inserted in (7) to make the magnitudes

Received May 2, 1983; revised January 9, 1984.

1980 Mathematics Subject Classification. Primary 65D20; Secondary 33A20, 41A50, 78A50.

Key words and phrases. Rational Chebyshev approximations, Fresnel integrals. 
TABLE I

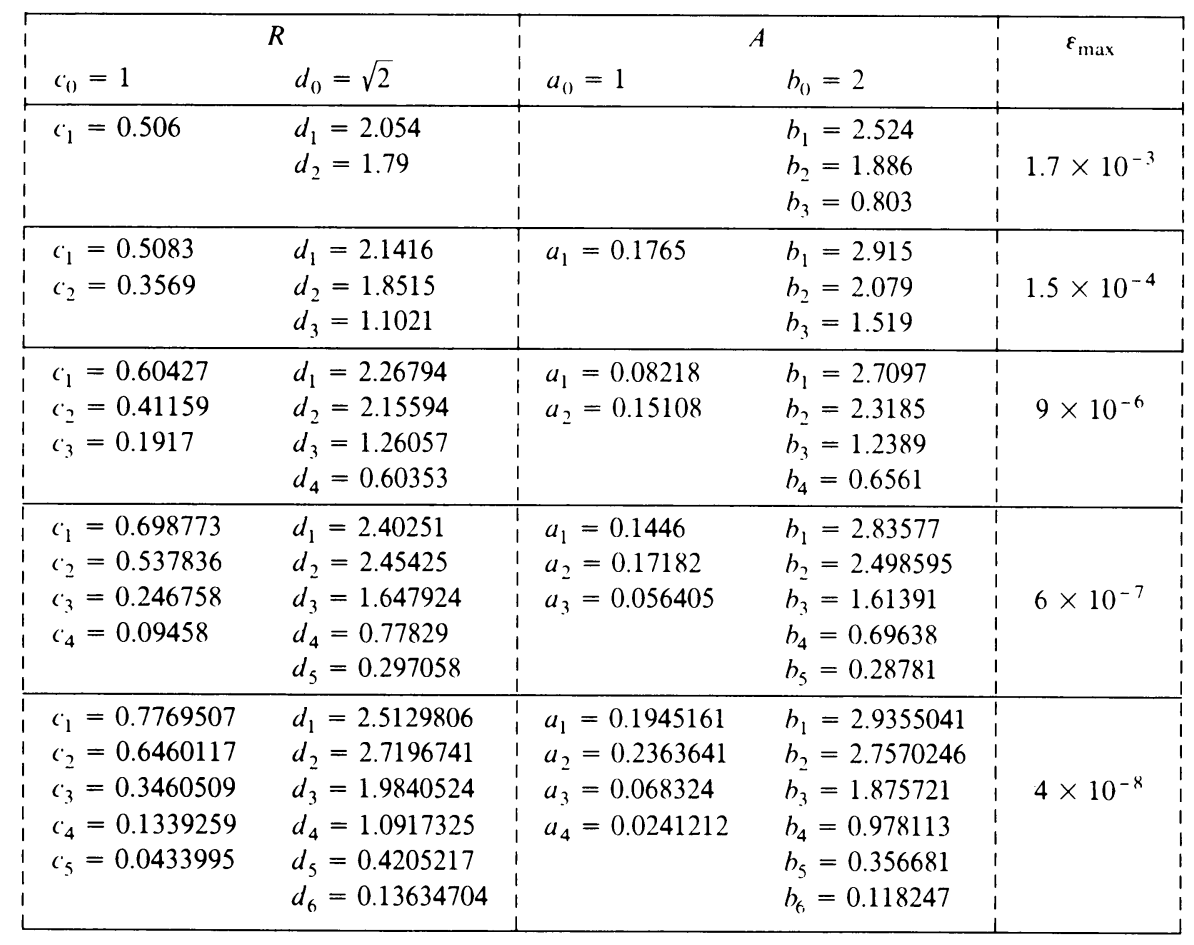

comparable. An overall index of error is the Pythagorean sum

$$
\varepsilon=\left[(\delta R)^{2}+(\delta A)^{2}\right]^{1 / 2}
$$

The error function $\varepsilon(x)$ of $(8)$ is therefore the diagonal distance between approximated and exact points in the Cornu $(C, S)$ plane.

We force these errors to go asymptotically to zero as $x \rightarrow 0$ by using fixed values of the coefficients $c_{0} / d_{0}=1 / \sqrt{2}$ and $a_{0} / b_{0}=1 / 2$. Likewise, the errors go asymptotically to zero as $x \rightarrow \infty$ for $m>l$ and $k>j$.

The coefficient values were determined by a numerical procedure, as follows. For an array of 100-plus "exact" data points $R_{0}(x)$ [for $A_{0}(x)$, independently] uniformly spanning the folded domain $0 \leqslant x \leqslant 1$ and $1>(1 / x) \geqslant 0$, computed from the Boersma subroutine, we used an iterative linearized least-squares routine to adjust crude initial values of the coefficients for a given choice of the indices $l, m$ [or $j, k$ ]. A subsequent routine located the $l+m+1$ [or $j+k+1]$ extrema of the error curve, computed the first derivatives of the extrema magnitudes with respect to each adjustable coefficient by numerical perturbation, and then solved for (linear) increments to the coefficient values that should equalize the extrema magnitudes. This routine was iterated a few times, as necessary, until the error curve (6) [or (7)] was indeed leveled in the minimax or Chebyshev sense. The Pythagorean error curve (8), computed for independent error curves (6) and (7) of comparable accuracy, has nearly leveled maxima.

This procedure was begun with the low-order cases (e.g., $l, m=1,2$ ), for which convergence difficulties were minimal. Adjusted coefficient values from one order 
were then used as input values for the initial least-squares adjustment of the next higher order. The final results were checked by recomputing the error curves using a subroutine based on the standard Maclaurin and asymptotic series, and by spot checks against the tables in Abramowitz and Stegun [1].

Table I lists the coefficients for five different orders of precision. Since all coefficients are positive, there are no difficulties with underflow. A number of other choices of order $l, m$ and $j, k$ were investigated, those listed in Table I being optimal in the sense of having the least error for a given number of adjustable coefficients. Graphs of the error functions (6)-(8) and listings of the APL computer routines are available from the author.

In the course of this work we recomputed Hastings' algorithm as given by Abramowitz and Stegun [1]. The precision is somewhat improved with revised coefficients as follows:

$$
\begin{gathered}
f(x)=\frac{1+0.882 x}{2+1.722 x+3.017 x^{2}}, \\
g(x)=\frac{1}{2+4.167 x+3.274 x^{2}+6.890 x^{3}} .
\end{gathered}
$$

The maximum (leveled) errors are $1.2 \times 10^{-3}$ and $1.0 \times 10^{-3}$ in the $f$ and $g$ functions, respectively. The maximum Pythagorean error in the Cornu plane, comparable to Eq. (8), is $2.2 \times 10^{-3}$ for Hastings' original coefficients and $1.6 \times 10^{-3}$ for our revision. Higher-order rational polynomials for the $f, g$ functions were found to give somewhat inferior precision compared to corresponding orders of polynomials for the $R, A$ functions.

This work was supported by Swarthmore College.

Department of Physics

Swarthmore College

Swarthmore, Pennsylvania 19081

1. M. Abramowitz \& I. A. Stegun, Editors, Handbook of Mathematical Functions, Dover, New York, 1965. See $\S \S 7.3 .32-33$ and Tables 7.7-8.

2. J. Boersma, “Computation of Fresnel integrals,” Math. Comp., v. 14, 1960, p. 380.

3. W. J. CoDy, "Chebyshev approximations for the Fresnel integrals," Math. Comp., v. 22, 1968, pp. $450-453$.

4. C. Hastings, JR., “Approximations for calculating Fresnel integrals,” Math. Comp. [MTAC], v. 10 , 1956, p. 173. 